\title{
Enquête
}

Archives de la revue Enquête

5 | 1997

Débats et controverses

\section{Les « variables lourdes » en sociologie électorale} État des controverses

Heavy variants in electoral sociology. A State of the Controversies

\section{Nonna Mayer et Daniel Boy}

\section{(2) OpenEdition}

Journals

Édition électronique

URL : http://journals.openedition.org/enquete/1133

DOI : 10.4000/enquete. 1133

ISSN : 1953-809X

Éditeur :

Cercom, Éditions Parenthèses

\section{Édition imprimée}

Date de publication : 1 septembre 1997

Pagination : 109-122

\section{Référence électronique}

Nonna Mayer et Daniel Boy, «Les « variables lourdes » en sociologie électorale », Enquête [En ligne], 5 | 1997, mis en ligne le 15 juillet 2013, consulté le 10 décembre 2020. URL : http://

journals.openedition.org/enquete/1133; DOI : https://doi.org/10.4000/enquete.1133 


\title{
Les « variables lourdes » en sociologie électorale
}

\author{
État des controverses \\ Heavy variants in electoral sociology. A State of the Controversies
}

\author{
Nonna Mayer et Daniel Boy
}

1 Y a-t-il des modèles en sociologie politique ? La classique notion de paradigme, telle qu'elle a été définie à l'origine par Thomas $\mathrm{S}$. $\mathrm{Kuhn}^{1}$, comme un ensemble de théories, de concepts, de notions de base qui, à un moment donné de l'histoire d'une discipline, constituent le cadre général de référence du travail scientifique pour une communauté de chercheurs, pourrait-elle, par exemple, s'appliquer à notre discipline ? À travers cette grille de perception les membres de cette communauté s'accordent sur un certain nombre de résultats acquis et sur les voies dans lesquelles la recherche doit se poursuivre, bref sur les questions qui doivent être posées à la réalité et sur les procédures qui doivent être mises en œuvre pour répondre à ces interrogations. Pour Kuhn, la découverte répétée d'anomalies, c'est-à-dire de faits qui contredisent la théorie en vigueur, affaiblit peu à peu le paradigme dominant et donne naissance, souvent parmi des chercheurs se situant en marge de la communauté, par exemple en raison de leur jeune âge ou de leur faible position institutionnelle, à un nouveau paradigme qui prétend rendre compte plus exactement des anomalies constatées. Une concurrence s'établit alors parfois entre l'ancien et un nouveau paradigme et la crise se résout normalement par la prééminence du second sur le premier mais non sans un certain nombre de retards et de contretemps.

2 Retracer les controverses qui se sont développées depuis une trentaine d'années dans l'explication du comportement électoral donne effectivement parfois le sentiment que l'existence de paradigmes concurrents rend compte de façon assez parlante du débat qui a eu lieu. On peut schématiquement distinguer trois paradigmes. Le premier, appelons-le "modèle sociologique ", est associé aux travaux pionniers du sociologue Paul Lazarsfeld et de son équipe, à l'Université de Columbia. Lors de l'élection présidentielle de 1940, qui oppose le républicain Willkie au démocrate Roosevelt, ils se proposent d'étudier l'effet de la campagne sur les choix électoraux. Un panel 
représentatif des habitants d'un comté de l'Ohio est interrogé tout au long de celle-ci à sept reprises. À leur grande surprise, la campagne n'a eu qu'un effet limité sur leurs choix politiques. Les électeurs se sont en majorité décidés bien avant la campagne et sont restés fidèles à leur choix initial, leurs orientations politiques sont stables et conformes aux normes de leur milieu familial, social et culturel. Inversement, la connaissance des groupes auxquels appartiennent les individus permet de prédire leur vote, comme le montre un indice de prédisposition politique combinant le statut social, la religion et le lieu de résidence. Les électeurs ruraux, protestants et aisés votent dans la proportion de trois sur quatre pour le candidat républicain, tandis que les électeurs urbains, catholiques et socialement défavorisés votent dans la même proportion pour le candidat démocrate. «Une personne pense, politiquement, comme elle est socialement. Les caractéristiques sociales déterminent les caractéristiques politiques ${ }^{2} »$ : telle est la principale conclusion de leur étude, qui démolit le mythe de la toute-puissance des médias tout comme celui d'un citoyen éclairé, parfaitement informé sur les candidats et les enjeux de la campagne.

Ce déterminisme social est sévèrement critiqué par les chercheurs du Survey Research Center de l'Université du Michigan qui font l'hypothèse d'un second modèle " psychopolitique ». Pour eux, le vote est d'abord un acte politique, commandé par la perception qu'ont les électeurs des principaux objets politiques. Ils s'appuient pour le démontrer sur les enquêtes nationales menées au SRC à partir de 1948 à l'occasion de chaque élection présidentielle, auprès d'échantillons représentatifs de la population en âge de voter, interrogés avant et après l'élection. Le comportement électoral est analysé comme la résultante d'un champ de forces psychologiques, qu'ils mesurent au plus près de l'élection considérée, en s'attachant surtout à explorer les attitudes des électeurs à l'égard des candidats, des partis et des programmes. La variable-clé du vote à leurs yeux est " l'identification partisane », attachement affectif et durable de l'électeur à un des deux grands partis qui structurent la vie politique américaine. Elle fonctionne comme un écran perceptif, filtrant la vision du monde des électeurs. Plus ils s'identifient à un parti, plus ils sont favorables aux candidats et aux positions qu'il soutient. La majorité des électeurs apparaît peu informée et peu intéressée par les questions politiques, incapable d'une réflexion abstraite et idéologique. Mais ils ont comme points de repère leurs lunettes partisanes: "Comme l'acheteur d'une automobile qui n'y connaît rien aux voitures sinon qu'il préfère une marque donnée, l'électeur qui sait seulement qu'il est démocrate ou républicain réagit directement à son allégeance (partisane)³.» Celle-ci, généralement forgée dès l'enfance et transmise par les parents, renforcée par le milieu social et professionnel, confère une grande stabilité aux choix électoraux. La mobilité est un phénomène marginal, qui caractérise surtout les électeurs les moins instruits, les moins intégrés socialement et politiquement.

Ces deux modèles à leur tour vont être remis en cause et concurrencés par un troisième, celui de "l'électeur rationnel ». Dans les années soixante V. O. Key déjà critiquait la notion d'un électorat passif, prisonnier de la «camisole de force » des déterminants sociaux ou psychologiques, et soulignait sa capacité à porter un jugement, positif ou négatif, sur les sortants. Mais la critique la plus décisive est portée par les auteurs de The Changing American Voter ${ }^{4}$. Ils estiment que ces modèles sont dépassés et que l'électeur américain a changé. Les identités partisanes sont en crise. La proportion des "indépendants ", qui ne se reconnaissent dans aucun parti, est passée de $23 \%$ en 1964 à $40 \%$ en 1974, et même chez ceux qui déclarent encore une identité 
partisane, elle commande de moins en moins souvent leur vote. Comparés aux électeurs des années Eisenhower, ils manifestent plus d'intérêt aux enjeux de l'élection et la proportion d'«idéologues » tels que les définissait l'équipe de Michigan a doublé. Et ils ont plus souvent tendance à choisir les candidats selon leurs positions sur ces mêmes enjeux plutôt que sur des critères partisans, d'où le concept de «vote sur enjeux ».

Ces évolutions tiennent à l'arrivée sur la scène électorale de nouveaux électeurs jeunes de moins de 21 ans, femmes, Noirs -, sans affiliation partisane préexistante. Ils reflètent aussi un changement de la nature des enjeux, plus dramatiques et plus concrets que ceux des années soixante, qu'il s'agisse de la question raciale, de la lutte contre la drogue ou de la guerre au Vietnam. Ils tiennent enfin à des facteurs structurels, le passage de la société industrielle à une société postindustrielle, caractérisée par le gonflement du tertiaire et le déclin du secteur industriel, la hausse des valeurs «postmatérialistes» et la «mobilisation cognitive» des citoyens ${ }^{5}$. Plus instruits, mieux informés, ils seraient plus exigeants, plus autonomes, et à la recherche de formes alternatives de participation politique.

6 À la faveur de ces débats un modèle plus ancien, proposé par Anthony Downs dans son ouvrage classique, An Economic Theory of Democracy (1957), se trouve remis à l'honneur. L'électeur qu'il décrit est le frère jumeau de l'homo ceconomicus. Les acteurs politiques sont « rationnels » dans la mesure où ils cherchent à adapter les moyens aux fins qu'ils poursuivent. L'électeur-type ferait son choix sur le marché politique comme le consommateur qui achète une marque de lessive, il voterait au coup par coup pour le parti qui maximise son bénéfice ou son "utilité ${ }^{\star}$ ». Et sa perception des candidats en présence, de leurs promesses futures et de leurs réalisations passées, pèserait plus sur son choix que les affiliations partisanes ou les solidarités religieuses ou confessionnelles. Ce modèle lui-même est aujourd'hui très controversé aux États-Unis

7 Ces modèles ont fortement influencé l'analyse électorale outre-Atlantique et connu les mêmes vicissitudes. L'érosion des identifications partisanes, le recul de l'influence de la religion et de la classe sociale et la montée du « vote sur enjeux » touchent à des degrés divers la plupart des grandes démocraties occidentales ${ }^{8}$. En France en particulier, un pourcentage croissant d'électeurs a le sentiment que les notions de droite et de gauche, qui jouent ici le même rôle structurant que l'identification aux démocrates ou aux républicains aux États-Unis, sont "dépassées ${ }^{9}$ ». La volatilité accrue de l'électorat depuis 1981 et la montée des partis hors systèmes comme les Verts ou le Front national semblent confirmer ce brouillage des repères traditionnels. Comme aux États-Unis, elles conduisent à remettre en cause les modèles déterministes et à saluer la naissance de l'électeur "rationnel», alors que, précédemment, c'était principalement les économistes qui appliquaient au vote les modèles du choix rationnel ${ }^{10}$. Lors des élections législatives de 1988, Alain Lancelot et Philippe Habert en tracent le portrait suivant : « Moins contraint par le jeu des pesanteurs partisanes et idéologiques, rendu à son libre arbitre par la disparition progressive de structures d'encadrement traditionnelles, accédant aux logiques de l'individualisme électoral par le recours aux normes personnelles, le nouvel électeur affirme une autonomie croissante dans la prise de décision électorale et module ses choix à partir d'une adaptation stratégique aux variations de l'offre électorale et aux enjeux du scrutin ${ }^{11}$. » 


\section{La résistance du clivage gauche/droite}

8 L'élection présidentielle de 1995 offre l'occasion de tester la validité respective de ces modèles dans le cas français. Une enquête effectuée au lendemain du second tour montre que $98 \%$ des Français se placent sur l'échelle gauche/droite mise au point par la SOFRES ${ }^{12}$. Sur le long terme, la proportion des sans réponses a même baissé, passant de $10 \%$ en 1964 à $2 \%$ en 1995, de même que la proportion de ceux qui ne se situent ni à gauche ni à droite mais au centre, en position 4 (36 \% en 1964, $25 \%$ en 1995). Autrement dit les trois quarts des Français se définissent encore en 1995 comme de gauche ou de droite (Tableau 1).

Tableau 1. Autopositionnement sur l'échelle gauche/droite en 1995

\begin{tabular}{ccccccccc}
\hline GAUCHE & 1 & 2 & 3 & 4 & 5 & 6 & 7 & DROITE \\
\hline & $5 \%$ & $11 \%$ & $20 \%$ & $25 \%$ & $19 \%$ & $13 \%$ & $5 \%$ & \\
\hline
\end{tabular}

Question: «On classe habituellement les Français sur une échelle de ce genre qui va de la gauche à la droite. Vous, personnellement, où vous classeriez-vous sur cette échelle? »

Enquête cEVIPOF/sofres 1995, effectif 4038 (sans réponse 2\%).

Ce positionnement a une cohérence idéologique. Selon qu'ils se situent à gauche ou à droite sur cette échelle ils n'ont pas les mêmes valeurs. Leurs divergences les plus nettes, en 1995, portent sur le rôle de l'État dans la vie économique, la laïcité et l'immigration. $27 \%$ des électeurs les plus à gauche (cases 1 et 2 ) jugent les privatisations «très " ou « assez positives » contre $72 \%$ des électeurs les plus à droite (cases 6 et 7), 32 \% jugent positive l'école privée (contre $80 \%$ ) et $55 \%$ estiment qu'il y a trop d'immigrés en France (contre $93 \%$ ).

10 Ces choix idéologiques structurent enfin fortement leurs choix électoraux. Plus ils se situent à gauche sur l'échelle, plus ils sont nombreux à avoir voté au premier tour pour un candidat de gauche ou écologiste, dans une proportion qui varie de $86,5 \%$ à $92 \%$ (Tableau 2). Plus ils se situent à droite plus ils votent pour les candidats de la droite dans une proportion qui passe de $63 \%$ chez les centristes à 92 et $97 \%$ à mesure que l'on va vers la droite. On note toutefois que la proportion d'électeurs se situant à gauche et votant à gauche a baissé en moyenne de 6 points d'une élection présidentielle à l'autre, essentiellement au profit du vote Le Pen, deux fois plus fréquent chez eux qu'en 1988. Mais au second tour le clivage gauche/droite retrouve sa vigueur première (Tableau 2).

11 L'idée d'une disparition du clivage gauche/droite parmi les électeurs semble donc aujourd'hui bien mal étayée par les données d'enquête dont nous disposons. Mais cette théorie existe aussi sous une autre variante selon laquelle c'est moins la capacité des électeurs à se situer dans l'espace politique qui serait en question que leur aptitude à repérer les hommes politiques sur cette même dimension. Selon cette hypothèse, la convergence du discours des hommes politiques de gauche ou de droite sur les grands problèmes contemporains, en somme les conséquences de la « pensée unique » auraient pour effet une opacité croissante du champ politique: comment situer des acteurs politiques qui tiennent tous, peu ou prou, le même discours? 
Tableau 2. Vote aux élections présidentielles de 1995 selon la position sur l'échelle gauche/droite (\%)

\begin{tabular}{|c|c|c|c|c|c|}
\hline \multirow{2}{*}{$\begin{array}{l}\text { Position sur } \\
\text { l'échelle }\end{array}$} & \multicolumn{3}{|c|}{ Vote $1^{\text {er }}$ tour } & \multicolumn{2}{|c|}{ Vote $2^{\mathrm{e}}$ tour } \\
\hline & Gauche & Droite & $\begin{array}{r}\text { dont } \\
\text { LePen }\end{array}$ & Jospin & Chirac \\
\hline 1 & 90 & 10 & 7 & 97 & 3 \\
\hline 2 & 92 & 8 & 5 & 98 & 2 \\
\hline 3 & 86,5 & 13,5 & 6 & 92 & 8 \\
\hline 4 & 37 & 63 & 16,5 & 42,5 & 57,5 \\
\hline 5 & 8 & 92 & 12 & 7 & 93 \\
\hline 6 & 3 & 97 & 19,5 & 2 & 98 \\
\hline 7 & 3 & 97 & 37 & 7 & 93 \\
\hline
\end{tabular}

Enquête CEVIPOF/sofres 1995, non-votants et sans réponse exclus.

Lire : $97 \%$ de ceux qui se situentsur la première case de l'échelle gauche/droite ont voté Jospin au $2^{\mathrm{e}}$ tour.

Dans l'enquête menée par le CEVIPOF après l'élection présidentielle de 1995 on demandait aux personnes interrogées de situer les candidats à la présidentielle sur une échelle gauche/droite identique à celle que la personne utilise pour se classer ellemême. Or les résultats semblent indiquer que les personnes interrogées, contrairement à la théorie d'un espace politique "rétréci ", utilisent largement les possibilités de l'échelle gauche/droite pour classer les candidats de l'extrême gauche à l'extrême droite ${ }^{13}$.

\section{Le déclin limité des variables lourdes}

Les travaux pionniers de Michelat et Simon ${ }^{14}$ ont montré l'influence déterminante de la classe sociale et de la religion sur le vote. Dans les années soixante-dix, un clivage électoral majeur oppose le monde ouvrier, majoritairement déchristianisé, qui voit dans le parti communiste le défenseur naturel de la classe ouvrière, et vote en majorité pour la gauche, à celui des catholiques déclarés, principalement issu des classes moyennes rurales ou urbaines, acquis aux valeurs et aux partis de droite. Plus les individus ont d'attaches avec le milieu ouvrier, plus ils votent pour le PCF et pour la gauche. Plus ils pratiquent, plus ils sont à droite.

En 1995 comme en 1988, le degré d'intégration à la communauté catholique et à ses valeurs reste un élément décisif du choix électoral, au premier comme au second tour. La probabilité de voter pour un candidat de gauche diminue régulièrement avec la fréquence de la pratique religieuse, passant au premier tour de $81 \%$ chez ceux qui se déclarent sans religion à $30 \%$ chez les catholiques qui vont à la messe au moins une fois par mois et descendant jusqu'à $11 \%$ chez ceux qui y vont plusieurs fois par semaine, soit des écarts analogues à ceux observés en 1988.

En revanche, la spécificité du groupe ouvrier s'est progressivement estompée. Si le vote de gauche y reste majoritaire, l'écart avec les salariés non ouvriers a pratiquement disparu. Ce recul de la gauche est lié aux transformations du monde ouvrier, touché de plein fouet par les restructurations industrielles et le chômage. Il bénéficie aujourd'hui au Front national, qui au premier tour de l'élection présidentielle de 1995 a fait chez eux son meilleur score. À la faveur des déceptions suscitées par l'arrivée de la gauche au pouvoir, c'est lui qui apparaît aujourd'hui à beaucoup comme le défenseur privilégié 
des petits contre les gros, du peuple contre les élites ${ }^{15}$. Inversement les classes moyennes salariées se sont rapprochées de la gauche. Le Parti socialiste, rénové en 1971, attire tout particulièrement ces catégories jeunes, citadines, instruites, détachées de la pratique religieuse, sensibles aux valeurs post-soixante-huitardes et rebutées tant par l'ouvriérisme du PCF que par le conservatisme des partis de droite.

$\mathrm{Au}$ terme de ces évolutions le principal clivage socioprofessionnel reste celui qui oppose les « indépendants ", travaillant à leur compte, qui votent en majorité pour la droite, aux salariés, ouvriers ou non ouvriers, soumis aux ordres d'un patron, qui votent en majorité pour la gauche ${ }^{16}$. Médecins ou épiciers, agriculteurs ou chefs d'entreprise, ils sont propriétaires de leurs moyens de travail, attachés à la libre entreprise et à l'initiative individuelle. Ils se sentent menacés par une gauche qui leur paraît plus attentive aux droits des salariés, et plus favorable à l'intervention régulatrice de l'État dans la vie économique et sociale. 29 points d'écart séparent le niveau du vote pour la gauche de ces deux catégories en 1995, écart moins prononcé qu'en 1988 ou en 1978 (respectivement 33 et 38 points) mais encore appréciable.

Parmi les salariés un second clivage s'affirme, qui oppose le secteur privé au secteur public au sens large ${ }^{17}$. Qu'ils soient cadres, ingénieurs ou techniciens, employés de banque ou du commerce, contremaîtres ou techniciens, les salariés des entreprises privées votent majoritairement pour la droite. En revanche ceux du public votent beaucoup plus souvent pour la gauche, dans des proportions qui atteignent $69 \% \mathrm{chez}$ les professeurs, $64 \%$ chez les instituteurs et plus de $50 \%$ chez les autres salariés, l'écart entre le niveau du vote de gauche du privé et du public atteignant 21 points chez les cadres et les ingénieurs, et 14 points chez les ETAM (employés, techniciens, agents de maitrise). Déjà présent en 1978, ce clivage pourrait se renforcer à la faveur de la crise économique qui fait apparaitre les salariés du public comme des privilégiés, assurés de la sécurité de l'emploi et d'une meilleure retraite que les salariés du privé. Les attaques mêmes dont ils font l'objet et les inquiétudes liées à la mise en œuvre du traité de Maastricht, aux risques de déréglementation et d'atteinte au service public qu'il comporte, vont dans le même sens.

Le clivage gauche/droite structure encore les choix des Français, la religion et dans une moindre mesure la classe sociale pèsent sur la décision électorale. Qu'en est-il du modèle concurrent, celui de l'électeur rationnel?

\section{Le modèle de l'électeur rationnel}

Il se fonde sur l'hypothèse d'un électeur «rationnel» ou du moins doté d'une forte propension à se déplacer dans l'espace politique au gré des consultations électorales. Pour les tenants de cette théorie, les déterminants classiques du comportement électoral - proximités politiques ou variables de positions sociales - seraient devenus impuissants à expliquer les variations du vote. À chaque élection une frange croissante d'électeurs ferait son choix non plus en fonction de ses appartenances sociales et/ou politiques mais selon l'offre politique du moment et la conjoncture ${ }^{18}$, un peu comme un consommateur sélectionne, parmi des produits concurrents, celui qui satisfait le plus complètement ses exigences. Le profil de ce «nouvel électeur » correspondrait à celui de groupes sociaux cultivés et intéressés par la politique, dotés, par conséquent, d'une compétence politique propre à leur faire apprécier au cas par cas les «raisons» de choisir tel ou tel candidat ${ }^{19}$. 
20 En réalité la théorie de l'électeur rationnel est née de la constatation ou de la supposition d'une volatilité croissante des électeurs dans les démocraties modernes. On peut évidemment douter, comme l'ont remarqué certains auteurs ${ }^{20}$, que le simple fait qu'un électeur soit «mobile» entre deux tours d'élections implique qu'il soit plus rationnel. Mais c'est néanmoins ce problème de mobilité qui a polarisé l'attention des politologues, pour la simple raison qu'une forte mobilité paraissait plus ou moins mettre en question le paradigme déterministe.

Dans quelle mesure ce modèle trouve-t-il des confirmations dans les données empiriques? La première difficulté, pour étudier cet aspect du comportement électoral, réside dans le fait qu'il n'existe pas de définition universellement admise de la notion de "mobilité politique». Une définition stricte de la mobilité devrait conduire à considérer comme mobile tout électeur qui à deux tours d'élections différents ne reproduit pas son choix à l'identique. Mais la diversité des types d'élections et la fluidité relative du système partisan rendent à peu près impossible une définition aussi rigoureuse. Ainsi un vote aux élections législatives pour un candidat «divers gauche » ou "divers droite » ne trouve généralement pas son équivalent à l'élection présidentielle. De même les deux familles politiques qui composent en France la droite modérée, UDF et RPR, ne sont pas nécessairement représentées sur tout le territoire national aux élections que l'on cherche à mettre en perspective. En pratique on est donc nécessairement conduit, pour effectuer des comparaisons d'élection en élection, à regrouper les candidatures en familles politiques dotées d'une certaine stabilité au moins sur le moyen terme.

Convenons par exemple, pour comparer les votes aux élections législatives de 1993 et à la présidentielle de 1995, de regrouper partis et candidats en cinq catégories : extrême gauche et Parti communiste, gauche modérée, écologie, droite modérée et extrême droite, et retraçons à partir des déclarations des personnes enquêtées leurs trajets politiques (Tableau 3).

Tableau 3. Vote à l'élection présidentielle de 1995 selon le vote aux élections législatives de 1993 (\%)

\begin{tabular}{rrrrrrr}
\hline L 1993 & EG, PC & Gauche & Ecologie & Droite & ED & Effectif \\
\hline EG, PC & 58 & 29 & 2 & 6 & 5 & 218 \\
Gauche & 13 & 70 & 3 & 9 & 5 & 778 \\
Ecologie & 19 & 25 & 26 & 23 & 7 & 118 \\
\hline Droite & 2 & 5 & 1 & 81 & 11 & 1204 \\
E D & 2 & 2 & 1 & 10 & 86 & 119 \\
\hline
\end{tabular}

Enquête CEVIPOF/SOFRES 1995, non-votants et sans réponse exclus.

Lire : 5\% de ceux qui ont voté EG, PC en 1993 ont voté ED en 1995.

23 À partir de ce tableau, où situer les frontières de la mobilité et de la stabilité ? En considérant ceux qui ne votent pas pour la même famille politique aux deux élections on arrive à un total de $27 \%$ de mobiles. Mais une mobilité au sein de la famille de gauche (extrême gauche, gauche et écologie) ou au sein de la famille de droite (droite et extrême droite) doit-elle être considérée comme "identique " à un trajet de mobilité qui franchit la frontière gauche/droite, elle-même relativement arbitraire? Si l'on 
retient le critère du franchissement de cette limite symbolique, on ne trouve plus que $11 \%$ d'électeurs mobiles ${ }^{21}$.

Concentrons-nous sur les $11 \%$ d'électeurs qui passent de droite à gauche ou de gauche à droite entre les deux élections considérées puisque c'est bien pour l'essentiel la répartition gauche/droite que le modèle déterministe prétendait expliquer et que, d'une certaine façon, ces électeurs "échappent " à ce mode d'explication puisqu'ils passent la frontière gauche/droite. Quels sont les facteurs qui expliquent le fait d'être mobile plutôt que stable?

Dans le tableau 4 trois catégories ont été construites à partir des trajets politiques déclarés entre les législatives de 1993 et la présidentielle de 1995 : les stables au sein de la gauche, les stables au sein de la droite et les mobiles (de gauche à droite ou de droite à gauche). Ces catégories sont étudiées ici en fonction de quatre critères: le groupe socio-professionnel, la pratique religieuse, la position sur l'échelle gauche/droite et l'intérêt pour la politique.

Dans la première partie du tableau on voit que la religion rend compte parfaitement de la stabilité à gauche ou à droite mais ne fait pas varier le pourcentage de mobiles. Quant à la classe sociale, on retrouve la stabilité de l'orientation à droite des " indépendants ", mais on note la proportion relativement importante d'ouvriers "mobiles" (en détaillant le contenu de ce trajet on trouve bien sûr que cette mobilité des ouvriers se compose pour une bonne partie des trajets de la gauche vers le Front National). Les deux dernières parties du tableau 4 éclairent la signification de la mobilité politique mesurée. Le pourcentage de mobiles est maximum d'une part chez ceux qui se situent au centre de l'échelle politique (24\%) de l'autre parmi les électeurs qui déclarent un faible intérêt pour la politique.

Tableau 4. Stabilité et mobilité politique de 1993 à 1995 selon le groupe socio-professionnel, la pratique religieuse, la position sur l'échelle gauche/droite et l'intérêt pour la politique (\%)

\begin{tabular}{rrrrr}
\hline & $\begin{array}{r}\text { Stables } \\
\text { dans la Gauche }\end{array}$ & Mobiles & $\begin{array}{c}\text { Stables } \\
\text { dans la Droite }\end{array}$ & Effectif \\
\hline $\begin{array}{r}\text { Pratique religieuse : } \\
\text { Pratiquants réguliers }\end{array}$ & 16 & 10 & 74 & 369 \\
Pratiquants irréguliers & 25 & 11 & 64 & 439 \\
Non pratiquants & 43 & 12 & 45 & 1119 \\
Sans religion & 63 & 10 & 27 & 386 \\
\hline Groupe socio-prof.: & & & & \\
Indépendants & 18 & 8 & 74 & 379 \\
Salariés & 46 & 7 & 47 & 733 \\
Ouvriers & 41 & 15 & 44 & 1104 \\
\hline Position sur échelle: & & & & \\
Extrême gauche (1) & 88 & 8 & 4 & 162 \\
Gauche (2,3) & 85 & 10 & 5 & 758 \\
Centre (4) & 27 & 24 & 49 & 469 \\
Droite (5, 6) & 2 & 7 & 91 & 892 \\
Extrême droite (7) & 1 & 6 & 93 & 132 \\
\hline Intérêt politique: & & & & \\
Beaucoup & 49 & 5 & 46 & 369 \\
Assez & 41 & 8 & 51 & 1006 \\
Peu & 34 & 15 & 51 & 812 \\
Pas du tout & 30 & 20 & 50 & 271 \\
\hline
\end{tabular}

Enquête CEVIPOF/sofres 1995 (à l'exclusion des sans réponse pour chacun des indicateurs) 

déterministes? En fin de compte ce que l'on trouve ici ne va guère dans le sens de l'électeur «rationnel » tel qu'il était postulé par le paradigme évoqué, celui d'un électeur cultivé, intéressé par la politique, et «compétent ». D’autres tris qui ne sont pas reproduits ici nous indiquent au contraire que l'électeur mobile repéré par cette méthode a, contrairement au postulat de l'électeur rationnel, un niveau d'études, un revenu, un patrimoine moins élevés que la moyenne des électeurs. Ce profil correspond à un phénomène explicable aussi bien par le « modèle sociologique » que par le modèle " psycho-politique ». Il s'agit en réalité de cette catégorie d'électeurs décrite comme le " marais ${ }^{22}$ ", c'est-à-dire des électeurs peu informés politiquement et dont les allersretours entre la droite et la gauche proviendraient d'une incapacité à situer clairement les lignes de force du champ politique. Une autre explication pourrait aussi résider dans le fait que cette frange d'électeurs, peu intéressée par la politique, déclare pour cette raison même des orientations politiques «contradictoires" alors que le répondant intéressé par la politique cherche à donner à l'enquêteur une image de cohérence politique. Il existe enfin une troisième explication, triviale mais qui correspond de fait à ce que l'on observe de la réalité politique actuelle : les modèles d'explication déterministes prévoyaient à peu près correctement la répartition des électeurs en deux blocs. L'apparition d'une extrême droite dont la composante populiste est indéniable trouble sérieusement les modes d'explication traditionnels. Une fraction importante des mobiles que nous avons identifiés par cette méthode sont des électeurs des classes populaires qui rejoignent le vote en faveur de l'extrême droite au premier tour de la présidentielle, et dont une partie rejoint la gauche au second tour.

Le problème d'un modèle d'«électeur rationnel» concurrençant les modèles déterministes n'est pas pour autant réglé. Si l'on considère par exemple, non plus la mobilité entre gauche et droite mais la mobilité à l'intérieur du bloc de gauche, on trouve un groupe d'électeurs circulant entre vote socialiste, vote communiste et vote écologiste et dont le profil culturel correspond d'un peu plus près aux exigences du modèle de l'électeur rationnel. Ce phénomène est encore accru si l'on raisonne non plus en termes de reconstitutions de trajets électoraux mais à partir d'une question qui demandait aux personnes interrogées si elles avaient « sérieusement envisagé de voter pour un autre candidat (que celui qu'elles venaient d'indiquer à l'enquêteur) dans les deux ou trois mois précédant l'élection ». À cette question, $39 \%$ de ceux qui répondent indiquent effectivement un second choix possible. Or l'analyse de cette mobilité politique potentielle ou imaginaire est intéressante : là aussi on se rapproche sinon du modèle de l'électeur rationnel du moins de celui d'un électeur perplexe, hésitant souvent à l'intérieur de son camp (gauche ou droite) entre des candidats représentants des sensibilités différentes. Cet électeur se rapproche par certains traits de celui qui était prévu par le modèle de l'électeur rationnel : on trouve en particulier, parmi ceux qui ont hésité à l'intérieur du camp de la gauche, une proportion importante de personnes dotées d'un niveau d'études élevé et d'un intérêt politique supérieur à la moyenne. Autrement dit le modèle déterministe garde sa pertinence pour expliquer la répartition de l'électoral en deux blocs, entre la gauche et la droite. Mais aux marges du système on trouve des preuves empiriques, chez des électeurs instruits et politisés, d'un autre modèle explicatif du vote, où l'offre et la conjoncture électorale semblent avoir une influence accrue. 
Les deux modèles ne s'opposent donc pas, ils sont complémentaires. L'électeur n'est ni totalement libre, ni totalement déterminé, ni prisonnier des variables sociologiques, ni ballotté au gré de la conjoncture. Son choix est le fruit d'un processus où se mêlent facteurs sociaux et politiques, structurels et conjoncturels, à long terme et à court terme. La socialisation politique ne s'arrête pas avec l'enfance, c'est un phénomène cumulatif. Chaque individu appartient simultanément et successivement à une multitude de groupes aux sous-cultures spécifiques. Ces influences diverses et parfois contradictoires forment des sensibilités de droite ou de gauche. Quels que soient l'élection, ses enjeux, l'espace où elle se joue, les candidats en présence, les catholiques pratiquants seront plus conservateurs que les sans religion, les ouvriers plus à gauche que les patrons. Mais ces potentialités ne se réalisent que dans le cadre d'un scrutin particulier qui laisse place aux stratégies spécifiques des électeurs. Libre à eux de voter ou de ne pas voter, de participer aux deux tours ou à un seul, d'exprimer un vote ou de déposer dans l'urne un bulletin blanc ou nul, de préférer le candidat $\mathrm{A}$ ou le candidat $\mathrm{B}$, d'émettre un vote " pour » ou « contre », expressif ou instrumental.

\section{NOTES}

1. T. S. Kuhn, La structure des révolutions scientifiques, Paris, Flammarion, 1972.

2. P. F. Lazarsfeld, B. B. Berelson et H. Gaudet, The People's Choice, New York, Columbia University Press, 1944, p. 27. Voir aussi l'enquête suivante, dans la ville d'Elmira, qui approfondit la première et confirme globalement ses conclusions, B. B. Berelson, P. F. Lazarsfeld et W. N. McPhee, Voting, Chicago-Londres, University of Chicago Press, 1954.

3. A. Campbell, P. Converse, W. Miller et D. Stokes, The American Voter, New York, Wiley and Sons, 1960, p. 136.

4. V. O. Key Jr, The Responsible Electorate, Cambridge, Harvard University Press, 1966, et N. H. Nie, S. Verba et J. R. Petrocik, The Changing American Voter, Cambridge, Harvard University Press, 1979.

5. Ces transformations sont décrites en détail dans le livre de R. Inglehart, La transition culturelle dans les sociétés industrielles avancées, Paris, Economica, 1990.

6. Voir en particulier l'ouvrage de H. T. Himmelweit, P. Humphreys, M. Jaeger et M. Katz, How Voters Decide [1981], Philadelphie, Open University Press, 1986.

7. Pour un aperçu de cette controverse, déclenchée par la publication du livre de D. P. Green et I. Shapiro, Pathologies of Rational Choice Theory. A Critic of Applications in Political Science, New Haven, Yale University Press, 1994, voir en particulier le recueil dirigé par G. Friedman, The Rational Choice Controversy, Londres-New Haven, Yale University Press, 1996.

8. Pour un bilan comparatif voir M. Franklin, T. Mackie, H. Valen et al., Electoral Change. Responses to Evolving Social and Attitudinal Structures in Western Countries, Cambridge, Cambridge University Press, 1992.

9. 57 \% en décembre 1994 contre $33 \%$ en mars 1981. Voir SOFRES, L'État de l'opinion 1996, Paris, Seuil, 1996, p. 191.

10. En particulier les travaux de Jean Dominique Lafay, notamment « La théorie probabiliste du vote ", Revue d'Économie politique, 102, 1992, p. 487-518, et «Les interactions entre économie et politique », Journal de la Société de Statistique de Paris, 136, 1995, p.17-28. Voir également un numéro de la Revue française de Sociologie, 38, 1997, centré sur «L'économie du politique ", avec 
des contributions de R. Boudon, J. D. Lafay, A. Bernard, E. Dupoirier et N. Mayer. Pour un point de vue critique voir P. Merle, "L'homo politicus est-il un homo œconomicus? ", Revue française de Science politique, 40, 1990, p. 64-80, ainsi que celui des Américains D. P. Green et I. Shapiro, « Choix rationnels et politique. Pourquoi en savons-nous toujours si peu?», Revue française de Science politique, 46, 1995, p. 96-130. Pour une présentation des modèles économétriques, voir M. Servais, «Les modèles économétriques du vote", in N. Mayer, ed., Les modèles explicatifs du vote, Paris, L'Harmattan, 1997, p. 133-153.

11. «L'émergence d'un nouvel électeur? ", in P. Habert et C. Ysmal, eds, Les élections législatives de 1988, Paris, Le Figaro-Études politiques, 1988, p. 23.

12. Enquête du CEVIPOF administrée par la SOFRES après le second tour de l'élection présidentielle (9-20 mai 1995) auprès d'un échantillon national représentatif des Français inscrits sur les listes électorales $(\mathrm{N}=4$ 038). Sur l'évolution antérieure du clivage voir $\mathrm{G}$. Michelat, «A la recherche de la gauche et de la droite ", in CEVIPOF, L'électeur français en questions, Paris, Presses de la FNSP, 1990, p. 71-103.

13. La note moyenne (sur 7) variant de 1,9 pour A. Laguiller à 6,2 pour J.-M. Le Pen.

14. G. Michelat et M. Simon, Classe, religion et comportement politique, Paris, Éditions sociales, Presses de Sciences politiques, 1977.

15. Cf. N. Mayer, « Du communisme au Front national ", L'Histoire, 195, janvier 1996, p. 110-113, et P. Perrineau, «La dynamique du vote Le Pen : le poids du gaucho-lepénisme », in P. Perrineau et C. Ysmal, eds, Le vote de crise, Paris, Département d'Études politiques du Figaro et Presses de la FNSP, 1996, p. 243-263.

16. N. Mayer et E. Schweisguth, «Classe, position sociale et vote », in D. Gaxie, ed., L'explication du vote, Paris, Presses de la FNSP, 1985, p. 263-290.

17. Voir à cet égard F. de Singly et $\mathrm{C}$. Thélot, Gens du public, gens du privé. La grande différence, Paris, Dunod, 1988 ; D. Boy et N. Mayer, «Secteur public contre secteur privé : un nouveau conflit de classe », in N. Mayer, ed., Les modèles explicatifs du vote, op. cit., p. 111-132.

18. Voir par exemple, P. Merle, «L'homo politicus est-il un homo œconomicus ? ", op. cit.

19. C'est le modèle proposé par P. Habert et A. Lancelot, « L'émergence d'un nouvel électeur », in Les élections législatives de 1988, op. cit.

20. D. Boy et E. Dupoirier, «L'électeur est-il stratège?", in CEVIPOF, L'électeur français en questions, op. cit., p. 175-196.

21. En utilisant exactement la même méthode pour étudier les flux de mobilité entre les élections législatives de 1986 et présidentielle de 1988, on obtient respectivement les chiffres de $23 \%$ (mobilité au sens large) et de $9 \%$ (mobilité au sens étroit). Il y a donc une légère croissance de la mobilité électorale définie au sens large.

22. E. Deutsch, P. Weil et D. Lindon, Les familles politiques aujourd'hui en France, Paris, Minuit, 1966.

\section{RÉSUMÉS}

En science politique, trois modèles principaux d'explication des comportements électoraux sont en concurrence. Le premier, de nature sociologique, se fonde sur le postulat d'un effet des variables socio-économiques (sexe, âge, classe sociale, etc.). Le second prend appui sur une théorie de nature psycho-sociologique: l'électeur choisit son candidat à travers une identification partisane qui oriente son choix. Le troisième enfin, inspiré par des modèles 
économiques, suppose un électeur rationnel qui effectue des choix politiques en maximisant ses intérêts. Dans quelle mesure ces trois paradigmes d'explication rendent-ils compte des réalités du comportement électoral des Français depuis une vingtaine d'années?

In political science, three principal models of explaining electoral behaviours are in competition. The first, of a sociological nature, is based on the assumption of the effect of socio-economic variants (sex, age, social class, etc.). The second is based on a theory of a psycho-sociological nature: the elector chooses his candidate through a partisan identification which orients his choice. Finally the third, inspired by economic models, assumes a rational elector making his political choices while maximising his interests. To what extent do these three paradigms of explanation take account of realities of electoral behaviour of the French since some twenty years?

\section{AUTEURS}

\section{NONNA MAYER}

Nonna Mayer (CNRS) travaille dans le domaine de la sociologie électorale. Elle a notamment dirigé un ouvrage collectif sur Les modèles explicatifs du vote, Paris, L'Harmattan, 1997, un numéro de la Revue française de Sociologie centré sur « L'économie du politique » (XXXVIII-2,1997), ainsi que, avec Daniel Boy, un ouvrage sur l'évolution du comportement électoral des Français de 1978 à 1997 : L'électeur a ses raisons, Paris, Presses de Sciences politiques, 1997.

\section{DANIEL BOY}

Daniel Boy (Fondation nationale des sciences politiques) a participé aux enquêtes électorales du Centre d'études de la vie politique française en 1988 et 1995 ; il est coauteur, avec l'équipe électorale, d'ouvrages de sociologie électorale, en particulier : CEVIPOF, L'électeur français en questions, Paris, Presses de la FNSP, 1990 ; avec Nonna Mayer (sous la direction de), L'électeur a ses raisons, Paris, Presses de Sciences politiques, 1997. 